\title{
Extracellular ATP drives systemic inflammation, tissue damage and mortality
}

\author{
A Cauwels ${ }^{*, 1,2}$, E Rogge $^{1,2}$, B Vandendriessche ${ }^{1,2}$, S Shiva ${ }^{3}$ and P Brouckaert ${ }^{1,2}$
}

Systemic inflammatory response syndromes (SIRS) may be caused by both infectious and sterile insults, such as trauma, ischemia-reperfusion or burns. They are characterized by early excessive inflammatory cytokine production and the endogenous release of several toxic and damaging molecules. These are necessary to fight and resolve the cause of SIRS, but often end up progressively damaging cells and tissues, leading to life-threatening multiple organ dysfunction syndrome (MODS). As inflammasome-dependent cytokines such as interleukin-1 $\beta$ are critically involved in the development of MODS and death in SIRS, and ATP is an essential activator of inflammasomes in vitro, we decided to analyze the ability of ATP removal to prevent excessive tissue damage and mortality in a murine LPS-induced inflammation model. Our results indeed indicate an important pro-inflammatory role for extracellular ATP. However, the effect of ATP is not restricted to inflammasome activation at all. Removing extracellular ATP with systemic apyrase treatment not only prevented IL-1 $\beta$ accumulation but also the production of inflammasome-independent cytokines such as TNF and IL-10. In addition, ATP removal also prevented systemic evidence of cellular disintegration, mitochondrial damage, apoptosis, intestinal barrier disruption and even mortality. Although blocking ATP receptors with the broad-spectrum P2 purinergic receptor antagonist suramin imitated certain beneficial effects of apyrase treatment, it could not prevent morbidity or mortality at all. We conclude that removal of systemic extracellular ATP could be a valuable strategy to dampen systemic inflammatory damage and toxicity in SIRS.

Cell Death and Disease (2014) 5, e1102; doi:10.1038/cddis.2014.70; published online 6 March 2014

Subject Category: Experimental Medicine

Systemic inflammatory response syndromes (SIRS) can be caused by infectious (sepsis) or sterile insults (e.g., trauma, burns, pancreatitis, hemorrhage, ischemia-reperfusion or chemically induced injury). They are characterized by socalled systemic cytokine storms, cell damage and death, tissue injury, and may ultimately result in multiple organ dysfunction syndrome (MODS) and death if not curbed in time. Despite numerous (successful) preclinical research investments and (unsuccessful) clinical trials, no mediatordirected treatment is available yet and sepsis has become the number one killer worldwide. ${ }^{1}$ Most of the trials so far focused on targeting single (early) pro-inflammatory mediators. More recently, it has been proposed that targeting more downstream detrimental pathways 'common' to most SIRS, such as mitochondrial dysfunction and/or microcirculatory failure, could be better therapeutic strategies. ${ }^{2,3}$ Furthermore, as the gut has repeatedly been described as the 'motor' of critical illness and MODS, maintaining or repairing its barrier function may be key to successful SIRS treatment. ${ }^{4}$ In addition, the bench-to-bedside problem might also be due to the fact that, traditionally, research has approached SIRS and MODS, which are intrinsically complex and nonlinear phenomena, through a reductionist and linear attitude. Alternatively, we may therefore need to focus on (nonlinear) variability indices as biomarkers and mouse-to-men translatable indications for patient stratification, disease progression or treatment response. ${ }^{5,6}$

The inflammatory response following either infection or sterile tissue injury involves several types of pattern recognition receptors (PRR), including Toll-like receptors (TLRs), NOD-like receptors (NLRs), RIG-I-like receptors (RLRs) and C-type Lectin receptors (CLRs). Although sepsis was originally believed to be caused by the presence of bacteria in the blood, the clinical signs are frequently presented by patients without a detectable source of infection. ${ }^{7}$ In addition, similar and overlapping signaling networks seem to be involved in propelling our body's exaggerated and mortal response against both infectious and sterile inflammation. ${ }^{8}$ Not only various microbial components are recognized by PRRs, but also many endogenous danger-associated molecular patterns (DAMPs), which are usually intracellular molecules that have reached the extracellular space, either actively or passively following cellular damage or death. These include

\footnotetext{
${ }^{1}$ Inflammation Research Center, VIB, Ghent, Belgium; ${ }^{2}$ Department of Biomedical Molecular Biology, Ghent University, Ghent, Belgium and ${ }^{3}$ Department of Pharmacology and Chemical Biology and Vascular Medicine Institute, University of Pittsburgh School of Medicine, Pittsburgh, PA, USA

*Corresponding author: A Cauwels, VIB-UGent Department of Medical Protein Research, Albert Baertsoenkaai 3, B-9000 Ghent, Belgium. Tel: +32 92649287 ; Fax: +32 9 2649490; E-mail: anje.cauwels@ vib-ugent.be

Keywords: ATP; apyrase; suramin; LPS; in vivo

Abbreviations: AB/PAS, alcian blue/periodic acid-Schiff; CLRs, C-type lectin receptors; DAMPs, danger-associated molecular patterns; LDH, lactate dehydrogenase; LPS, lipopolysaccharide; MODS, multiple organ dysfunction syndrome; MPO, myeloperoxidase; NLRs, NOD-like receptors; PRR, pattern recognition receptors; RLRs, RIG-I-like receptors; SIRS, systemic inflammatory response syndromes; TLRs, Toll-like receptors; TUNEL, terminal deoxynucleotidyl-transferase-mediated deoxyuridine triphosphate nick-end labeling

Received 04.12.13; revised 23.1.14; accepted 27.1.14; Edited by M Agostini
} 
proteins, such as heat shock proteins or the chromatinassociated high-mobility group box 1 (HMGB1), and also (mitochondrial) DNA and purine metabolites such as uric acid, ATP and adenosine. ${ }^{9}$ The most common Gram-negative constituent is lipopolysaccharide (LPS), signaling via TLR4. When injected into mammals, including humans, LPS causes systemic inflammation and mimics most of the septic effects such as fever or hypothermia, tachypnea, tachycardia, cardiovascular collapse and organ dysfunction. ${ }^{10,11}$

Autocrine and/or paracrine signaling of extracellular ATP for the inflammatory activation of macrophages has been well established in vitro and suggested to be important in vivo as well. For studies of the NLRP3 inflammasome and the subsequent production of mature IL- $1 \beta$ and -18 , treatment of macrophage cultures with both LPS and ATP has even become the standard working model. ${ }^{12,13}$ During systemic inflammation, ATP may be released by activated platelets and leukocytes. In addition, also parenchymal cells such as epithelial or endothelial cells may exhibit regulated ATP release. ${ }^{14}$ This extracellular ATP can activate both $P 2 X$ and $\mathrm{P} 2 \mathrm{Y}$ purinergic receptors, and the $\mathrm{P} 2 \mathrm{X} 7$ receptor was originally thought to be specifically responsible for ATP-aided inflammasome activation in leukocytes, both in vitro and in vivo. ${ }^{15,16}$ However, more recently the involvement of the ATP-P2X7 axis for LPS-induced inflammasome activation in vivo has been questioned again. ${ }^{16,17}$ Under homeostatic conditions, extracellular ATP is removed by conversion into adenosine in a two-step enzymatic process involving the ectonucleotidases CD39 (ecto-apyrase) and CD73. However, under pathological conditions, including inflammation and hypoxia, extracellular ATP levels may increase due to active release as well as passive leakage from damaged or dying cells, in combination with downregulation of ectonucleotidases. ${ }^{18}$

In this paper, we suggest an important pro-inflammatory and toxic role for extracellular ATP, which is not restricted to inflammasome activation. We found that removal of extracellular ATP by apyrase treatment not only prevented systemic IL-1 $\beta$ accumulation but also precluded the induction of inflammasome-independent cytokines, such as TNF and IL-10, of mitochondrial damage, cellular disintegration, apoptotic death, intestinal injury and barrier breakdown, and subsequent mortality, in a murine model of LPS-induced shock. In contrast to other published studies, we chose to antagonize extracellular ATP signaling by removing extracellular ATP via apyrase treatment rather than using P2X7deficient mice or the currently available (broad-spectrum) P2 receptor antagonists, because recent evidence excludes a crucial role for P2X7 in LPS-induced IL-1 $\beta$ production in vivo ${ }^{17}$ and even suggests an important protective role for other P2X receptors. ${ }^{19}$ Indeed, although removal of extracellular ATP using apyrase successfully dampened various aspects of systemic LPS-induced inflammation, including mortality, both in pre- or post-treatment, the inhibition of $\mathrm{P} 2$ purinergic receptors with suramin could not protect and even exacerbated the systemic effects of LPS when given as a posttreatment, which may be due to aspecific effects of suramin and/or protective effects of particular P2 receptors. In addition, our results also exclude a critical role for P2 receptors in LPS-induced IL-1 $\beta$ production in vivo.
In conclusion, our data suggest a key role for extracellular ATP in triggering various aspects of systemic inflammation and cellular and tissue damage during endotoxemia, which may be successfully and completely antagonized by ATP removal but not by broad-spectrum blocking of its P2 receptors.

\section{Results}

Removal of extracellular ATP with apyrase dose dependently protects against LPS-induced hypothermia and mortality. To evaluate the contribution of extracellular ATP to inflammation in an in vivo setting, we used the ATP hydrolase apyrase in LPS-induced shock. Pretreatment with increasing doses (from 5 to $15 \mathrm{IU}$ per mouse) increasingly protected mice against a lethal challenge with LPS (Figures 1a and b). Next, we used 15IU apyrase to determine the capacity of apyrase to protect as a therapeutic post-treatment. As shown in Figures 1c and d, apyrase also protected significantly when given $30 \mathrm{~min}$ after the LPS challenge. However, when given $2 \mathrm{~h}$ after challenge, apyrase could no longer protect (not shown).

Extracellular ATP is necessary for systemic cytokine storm induction and inflammation. Using both genetic and therapeutic approaches, we recently determined the critical involvement of both endogenous IL-1 $\beta$ and IL-18 in several models of inflammatory and septic shock. ${ }^{20}$ To induce IL-1 $\beta / \mathrm{IL}-18$ maturation and release, the NLRP3 inflammasome is essential, and LPS-induced activation of the inflammasome has been suggested to result from particle internalization and/or ATP signaling. ${ }^{21}$ Hence, we decided to analyze the effect of apyrase on LPS-induced cytokine induction. As anticipated based on our hypothesis, IL-1 bioactivity and IL-1 $\beta$ protein levels in serum were significantly reduced (Figures $2 a$ and $b$ ). Surprisingly, however, we found that also endogenous TNF and IL-10 production were markedly inhibited by apyrase treatment (Figures $2 \mathrm{c}-\mathrm{e}$ ), whereas IL-6 levels were not affected significantly (Figure 2f). Also the production of IL-12 (produced from $2 \mathrm{~h}$ on) and IFN- $\gamma$ (undetectable at $2 \mathrm{~h}$, but systemically present at $6 \mathrm{~h}$ ), pro-inflammatory cytokines that may link innate with adaptive immunity, was reduced thanks to apyrase treatment, albeit not significantly (not shown). The reduced systemic TNF and IL-1 production was reflected in reduced leukocyte infiltration into liver tissue (Figure 3a).

Apyrase treatment reduces cellular disintegration, mitochondrial dysfunction and intestinal barrier breakdown. Intracellular proteins such as hexosaminidase, a lysosomal enzyme, are indicative for cellular damage and (necrotic) disintegration when detected systemically. As illustrated in Figure $3 b$, hexosaminidase release into the circulation was significantly attenuated by apyrase. Similarly, tissue breakdown demonstrated by increased lactate dehydrogenase (LDH) serum levels $6 \mathrm{~h}$ after LPS was dampened by apyrase treatment (not shown). In addition, apyrase also protected mitochondrial complex I and II activity in the liver (Figures 3c and d). In the small intestine, apyrase completely prevented apoptotic death of epithelial cells, as evidenced by 
a
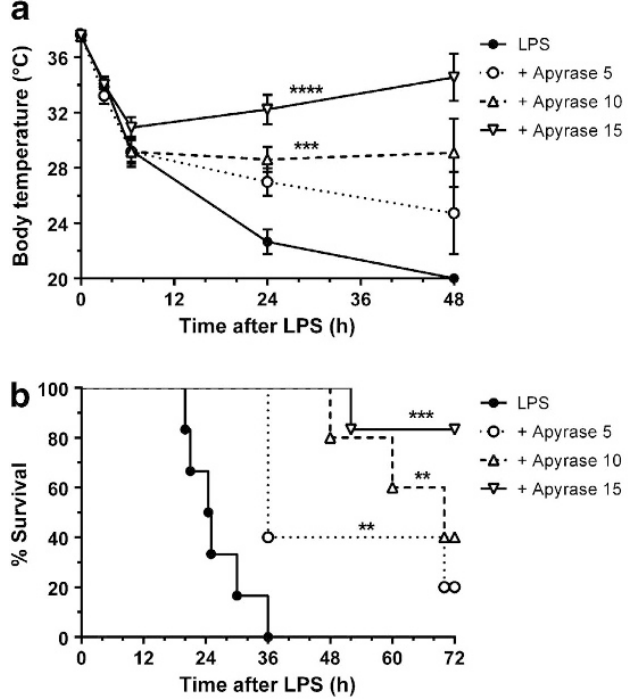
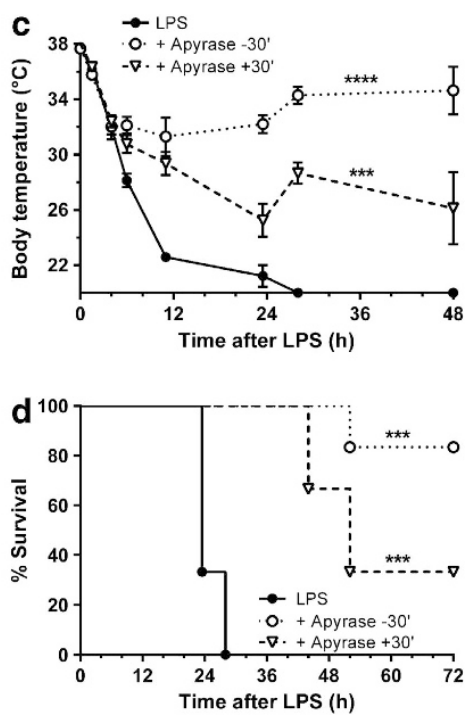

Figure 1 Apyrase dose dependently protects against LPS-induced shock. (a, b) Mice were injected i.v. with $175 \mu \mathrm{g}(8 \mathrm{mg} / \mathrm{kg})$ Sigma LPS, and pre-treated i.p. with 5, 10 or $15 \mathrm{U}$ apyrase 30 min before. ${ }^{* \star * \star} P<0.0001,{ }^{* \star *} P<0.001$, compared with LPS controls, $n=6$. (c, d) Mice were injected i.v. with $175 \mu \mathrm{g}$ Sigma LPS, and treated i.p. with $15 \mathrm{U}$ apyrase 30 min before or after LPS challenge. ${ }^{* \star \star *} P<0.0001,{ }^{* \star *} P<0.001$, compared with LPS controls, $n=6$
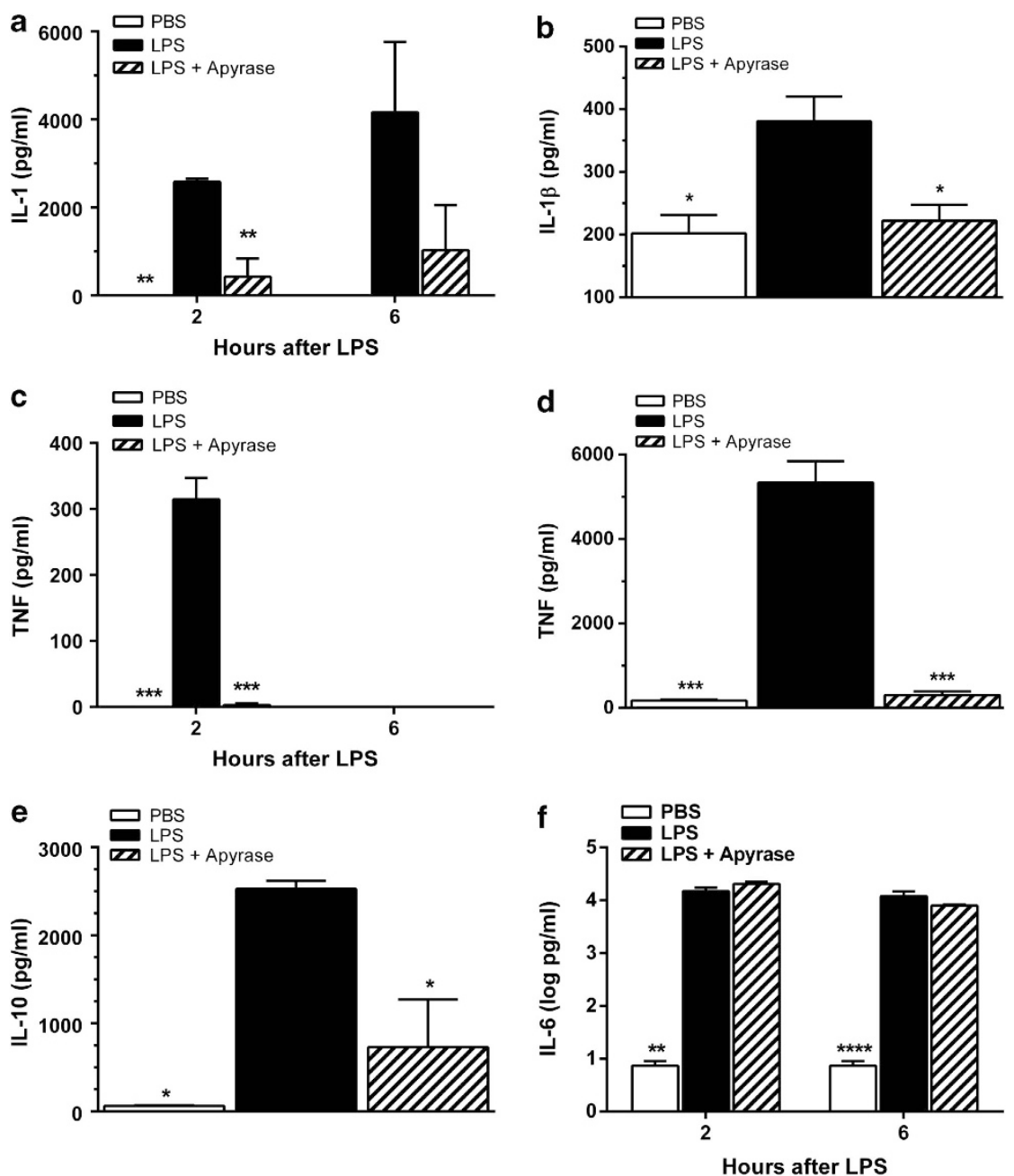

Figure 2 Apyrase inhibits systemic TNF, IL-1 and IL-10, but not IL-6 production. (a, c) Systemic IL-1 and TNF induction determined by bioassay ( $n=4)$. ${ }^{* \star} P<0.01$, ${ }^{* * *} P<0.001$, compared with LPS alone. (b, d, e) Serum IL-1 $\beta$, TNF and IL-10 levels $2 \mathrm{~h}$ after LPS challenge, determined by Bio-Plex $(n=3)$. (f) IL-6 concentrations in serum, assayed via Bio-Plex. ${ }^{*} P<0.05,{ }^{* \star *} P<0.001,{ }^{* * \star} P<0.0001$, compared with LPS alone 
a
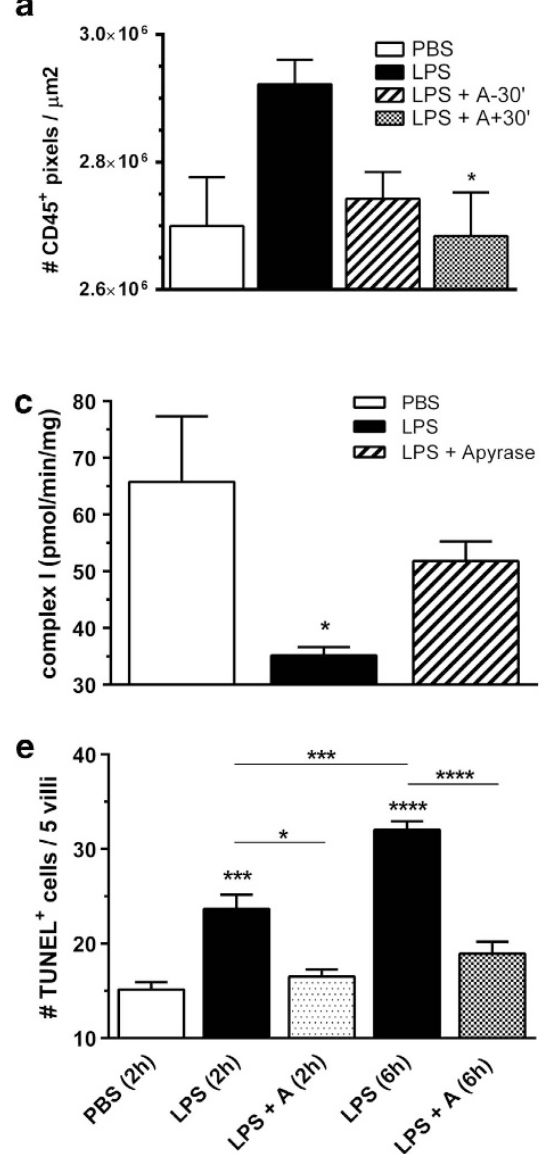
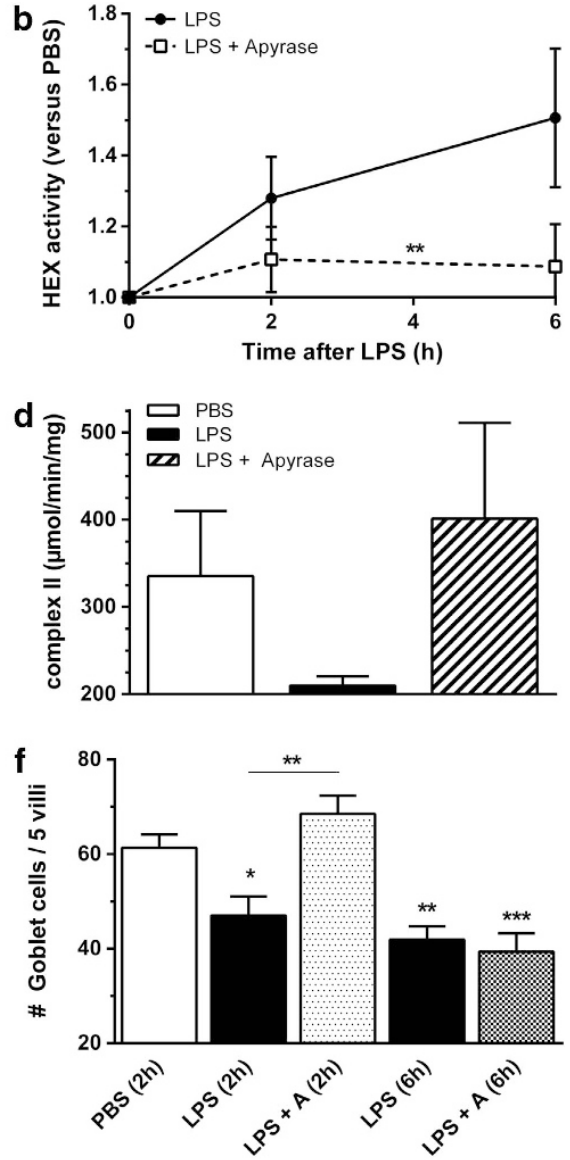

Figure 3 Apyrase prevents inflammation and tissue damage. (a) CD45 staining quantification in liver sections $6 \mathrm{~h}$ after LPS challenge, $n=3-7$. (b) Hexosaminidase in serum indicates cellular disintegration. Plotted is the relative increase compared with PBS-treated animals, $n=7$. (c, d) Mitochondrial complex I and II activity in liver tissue $6 \mathrm{~h}$ after LPS injection, $n=3-4$. (e) TUNEL staining of apoptotic cell death in the jejunum, $n=4-6$. (f) AB/PAS staining was used to visualize mucin-positive goblet cells in the jejunum, $n=6-9$. ${ }^{\star} P<0.05,{ }^{* \star} P<0.01,{ }^{* \star \star} P<0.001$, ${ }^{\star \star \star \star} P<0.0001$, compared with LPS (a, b) or PBS controls (c-f), or as indicated by horizontal lines

TUNEL staining (Figure $3 e$ ), as well as the early disappearance of mucin-producing goblet cells (Figure $3 f$ ).

\section{Removal of extracellular ATP is superior over blocking ATP receptors for protection. ATP is sensed by both $P 2 X$ and P2Y purinergic receptors, which may be blocked by the broad-spectrum antagonist suramin. When given as a pre- treatment, suramin efficiently prevented LPS-induced TNF production, leukocyte infiltration and necrotic tissue damage (Figures $4 \mathrm{a}-\mathrm{C}$ ). Nevertheless, suramin could not prevent IL-1 $\beta$ production (Figure $4 d$ ) or protect against mortality (Figure 4e). Surprisingly, when given as a post-treatment, suramin even accelerated mortality (Figure 4e), which was accompanied by increased levels of circulating TNF and $\mathrm{IL}-1 \beta$, as well as evidence of early tissue damage and exacerbated neutrophil infiltration (Figures $4 d$ and $f-h$ ).}

\section{Discussion}

Our results show that extracellular ATP is an important mediator of inflammation-associated systemic damage and toxicity in vivo. To study the possible involvement of ATP release, we used a model of LPS-induced systemic inflammation in mice. Removing extracellular ATP by means of the ATPase apyrase significantly protected against mortality, even when given as a post-treatment. This protection correlated with markedly reduced production of both the pro-inflammatory and cell death inducing cytokines TNF and IL-1, and the anti-inflammatory cytokine IL-10. Although apyrase protected as a post-treatment when given 30 min after LPS, there was no protective effect when given $2 \mathrm{~h}$ after challenge. This is not surprising, as both TNF and IL-1 are 'early' pro-inflammatory and toxicity-inducing cytokines, markedly increasing (and in case of TNF even peaking) within the first $2 \mathrm{~h}$ after LPS. ${ }^{17}$ Extracellular ATP had already been suggested to be an essential trigger for activation of the IL- $1 \beta$ producing caspase- 1 in the multiprotein inflammasome complex in macrophages ${ }^{21}$ but our current results clearly show that in an in vivo setting ATP signaling is involved in the production of other cytokines as well.

We have recently documented the critical involvement of RIPK-dependent necroptosis (also coined 'regulated necrosis', to distinguish it from accidental necrosis) in the release of endogenous DAMPs and subsequent mortality induced by either TNF or murine septic shock, ${ }^{22}$ as well as the crucial involvement of both IL-1 $\beta$ and IL-18 in several models of 

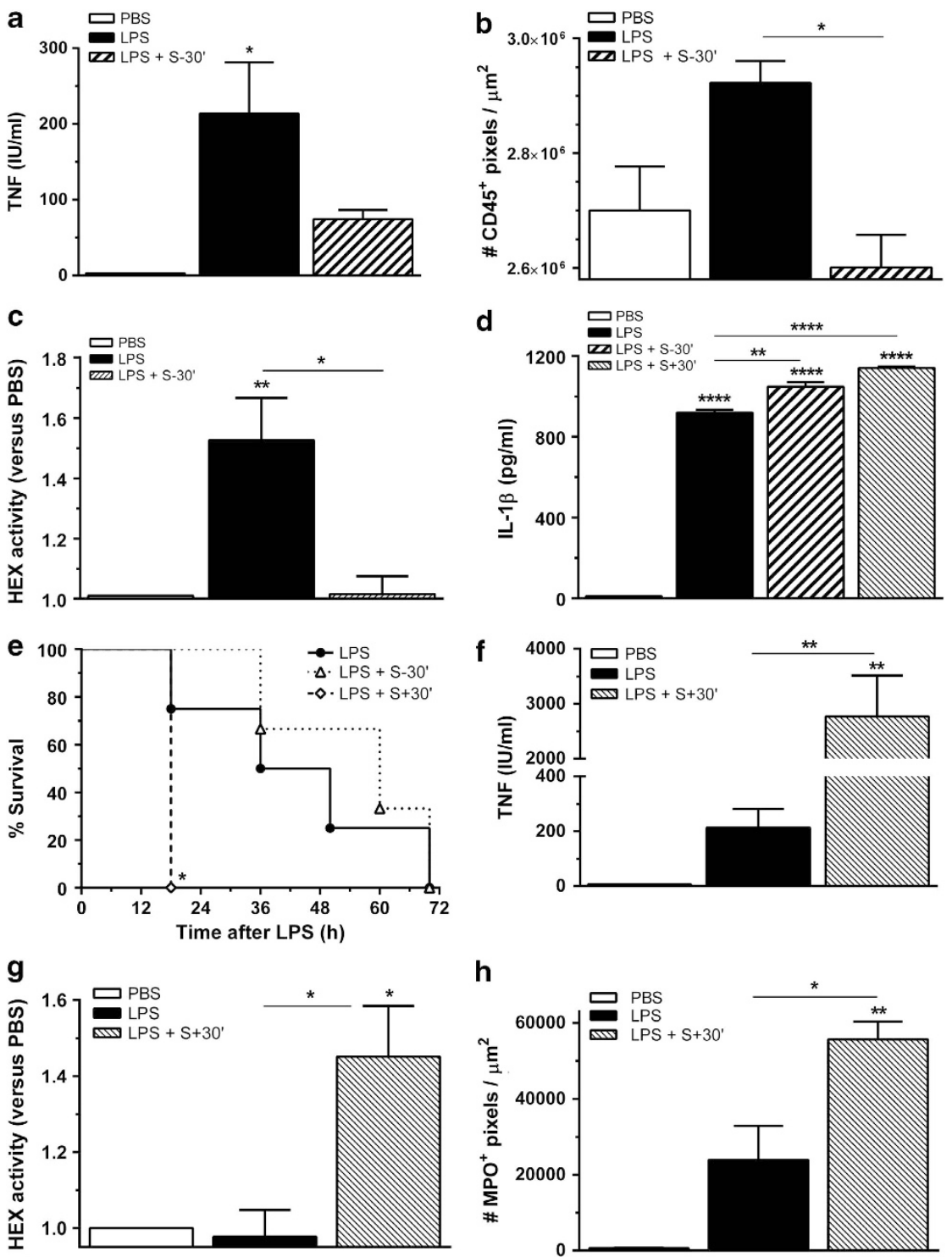

Figure 4 Suramin reduces inflammation and necrotic damage, but offers no survival benefit. (a-c) Suramin pre-treatment at -30 min reduces systemic TNF ( $2 \mathrm{~h}$ ), CD45 staining in the liver ( $6 \mathrm{~h}$ ) and systemic hexosaminidase activity $(6 \mathrm{~h}), n=3$. (d) Suramin does not reduce systemic IL-1 $\beta$ levels when given 30 min before or after LPS, rather contrary (2h), $n=3$. (e) In contrast to apyrase, suramin does not prevent LPS-induced mortality. When given as a post-treatment $(+30$ min), suramin even accelerates mortality (e), correlated with increased TNF production ( $\mathbf{f}, 2 \mathrm{~h}$ ), earlier evidence of cellular damage $(\mathbf{g}, 2 \mathrm{~h})$ and enhanced myeloperoxidase (MPO) accumulation in the liver (h, $6 \mathrm{~h}$ ). ${ }^{*} P<0.05,{ }^{* \star} P<0.01,{ }^{* \star \star *} P<0.0001$, compared with PBS controls (except for e), with LPS (only for e), or as indicated by horizontal lines

inflammatory and septic shock. ${ }^{20}$ In our current study, apyrase successfully prevented systemic evidence of LPSinduced necrotic and mitochondrial damage as well, most probably thanks to its remarkable effect on both endogenous TNF and IL-1. In addition, preventing caspase- 1 activation may also contribute to reducing inflammatory cell death. ${ }^{23}$

For decades already, the gut has been proposed as the 'motor' of several critical illnesses, driving MODS and mortality. ${ }^{4}$ The intestinal barrier, necessary to protect against microbiota and their toxins, consists of both a physical and an immunological part. The physical section is composed of a tightly sealed epithelial lining, reinforced by a thick mucus layer produced by goblet cells. Early after a systemic inflammatory insult, enterocytes undergo apoptosis, and mucin-positive goblet cell loss is observed. Treatment with apyrase completely prevented enterocyte apoptosis, which has been shown to be an important cause of increased permeability. This is most probably a reflection of the inhibitory effect of apyrase on systemic TNF production, ${ }^{24,25}$ possibly aided by its negative effect on IL- $1 \beta$ production. ${ }^{26}$ In addition, if mice were treated with apyrase, mucin-positive goblet cells started disappearing only at later time points. The exact mechanism of mucin- and/or goblet cell loss has not been elucidated yet, but it is generally believed that the formation of a thick mucus layer contributes significantly to intestinal barrier protection, providing a sterile zone and thus minimizing commensal bacterial contact with epithelial cells. ${ }^{27,28}$ All things considered, our data indicate that extracellular ATP removal may have a beneficial effect on intestinal barrier maintenance during inflammation, and consequently on the 
development and progression of SIRS and life-threatening MODS.

Remarkably, while apyrase protected as both pre- or posttreatment, the broad-spectrum P2 receptor antagonist suramin could not protect at all, despite inhibitory effects on TNF production, systemic evidence of cellular disintegration and inflammation, when given as a pre-treatment. However, in contrast to apyrase, suramin could not reduce systemic IL-1 $\beta$ levels, and, when injected as a post-treatment, suramin actually aggravated and/or accelerated TNF and IL-1 $\beta$ production, (necrotic) cellular damage and mortality. These results indicate that removing extracellular ATP is more successful than blocking $\mathrm{P} 2$ receptors to prevent systemic production/release of IL-1 $\beta$ in vivo. Despite numerous reports on the role of P2X7 receptors in ATP-mediated inflammasome activation and mature $\mathrm{IL}-1 \beta$ production in macrophages in vitro, its role in vivo has recently been questioned. ${ }^{17}$ Corroborating this, our results not only question the significant involvement of $\mathrm{P} 2 \mathrm{X} 7$ receptors in particular, but actually of $\mathrm{P} 2$ receptors in general, in the LPS-induced release of systemic IL-1 $\beta$. In addition, our data may also suggest either that ATP contributes critically to toxicity in a P2-independent manner or, and probably more likely, that certain P2 receptors may mediate beneficial effects during systemic inflammation. Many inflammatory mediators or pathways are known to possess two-sided Janus faces. Only recently, P2X1-deficient mice were shown to suffer more from LPS-induced morbidity due to exaggerated hemostatic disturbances and neutrophil accumulation. ${ }^{19}$ When given as a post-treatment, we found that suramin indeed augmented MPO infiltration, toxicity and morbidity. The reason why apyrase treatment does not affect the potentially protective P2 signaling pathway(s) may suggest that agonists other than ATP are involved in these protective effects. ${ }^{29}$ Alternatively, suramin may have detrimental side effects unrelated to ATP signaling, for instance by activating sarcoplasmic reticulum calcium release via the stimulation of ryanodine receptors ${ }^{30}$ or by its positive effect on tyrosine phosphorylation-dependent signaling. ${ }^{31}$

Taken together, our data suggest that systemic removal of extracellular ATP could be a better strategy than preventing ATP from signaling through its P2 purinergic receptors to dampen systemic inflammatory cell and tissue damage and toxicity. Removing extracellular ATP with apyrase very efficiently prevented the production of the highly inflammatory and destructive cytokines TNF and IL-1, which correlated with reduced leukocyte infiltration, mitochondrial and tissue damage, and destruction of the vital gut barrier. As both proinflammatory cytokines and leukocyte extravasation are strictly essential to kill and eliminate microbes, ATP removal should only be considered in case of sterile inflammation or in combination with broad-spectrum antibiotics.

\footnotetext{
Materials and Methods

Laboratory animals. Female C57BL/6J mice were purchased from Janvier (Le Genest St Isle, France) and housed in temperature-controlled, air-conditioned SPF facilities with 14/10 h light/dark cycles, food and water ad libitum. They were used at 10-16 wk. All experiments were approved by, and performed according to the guidelines of the animal ethics committee from Ghent University, Belgium, and the investigation conforms to the Directive 2010/63/EU of the European Parliament. For each experiment, mice were monitored several times daily for $5 \mathrm{~d}$. Moribund or surviving animals were euthanized by $\mathrm{CO}_{2}$ asphyxiation followed by cervical dislocation.
}

Reagents, injections and body temperature measurements. E. coli LPS (serotype 0111:B4), apyrase (grade VII from potato) and suramin sodium salt were purchased from Sigma (St. Louis, MO, USA) and injected in LPS-free PBS, intravenously for LPS, intraperitoneally for apyrase or suramin. Rectal body temperature was recorded with an electronic thermometer (model 2001; Comark Electronics, Littlehampton, UK).

Blood collection, $\mathrm{NO}_{x}^{-}$and cytokine measurements. Blood was collected from the tail vein or via cardiac puncture. Determination of $\mathrm{NO}_{2}^{-}+\mathrm{NO}_{3}^{-}$ $\left(\mathrm{NO}_{x}^{-}\right)$was done as described. ${ }^{32}$ For determination of TNF and IL-1 activity in serum, bioassays were performed. ${ }^{33}$ To corroborate the bioassay results, and to determine IL-6 and IL-10, Bio-Plex cytokine assay kits (Bio-Rad, Hercules, CA, USA) were used.

Determination of cellular and mitochondrial damage, and leukocyte infiltration. Hexosaminidase ${ }^{34}$ and lactate dehydrogenase (Cytotox96, Promega, Madison, WI, USA) activities were determined in serum. Whole-liver tissue, snap frozen in liquid nitrogen, was used for the measurement of mitochondrial complexes; for complex I, the rotenone sensitive rate of NADH oxidation was spectrophotometrically monitored at $340 \mathrm{~nm}$ in permeabilized tissue, whereas complex II activity was determined by measuring the reduction of dichloroindophenol at $600 \mathrm{~nm}$, coupled to the oxidation of $\mathrm{CoQ}_{2}$ using succinate as a substrate, and TTFA to determine specificity for complex II activity. Histopathology was evaluated on paraffin sections. In the jejunum, apoptosis was identified by the terminal deoxynucleotidyl-transferase-mediated deoxyuridine triphosphate nick-end labeling (TUNEL) technique using the ApopTag Peroxidase In Situ Apoptosis Detection kit (Millipore, Billerica, MA, USA); goblet cells were visualized using AB/PAS (Alcian blue combined with Periodic acid-Schiff) staining. To stain for leukocytes or neutrophils in liver sections, CD45 (30-F11, BD Pharmingen, Erembodegem, Belgium) or MPO (A-0398, Dako, Glostrup, Denmark) immunohistochemistry were performed.

Statistics. Statistics were performed using GraphPad Prism. Significant differences in systemic cytokines and $\mathrm{NO}_{x}^{-}$were examined using a one-way ANOVA with Bonferroni (for comparison of all pairs) or Dunnett (for comparison with a control group) post test. Data shown are always means \pm S.E.M. Two-way ANOVA was used to compare temperature or hexosaminidase curves (repeated measures), with Sidak's post hoc test for multiple comparisons. Survival curves were compared via a logrank test.

\section{Conflict of Interest}

The authors declare no conflict of interest.

Acknowledgements. We thank the animal care takers of our department. This work was supported by grants from the 'Fonds voor Wetenschappelijk Onderzoek (FWO)-Vlaanderen', the Interuniversity Attraction Pole (IAP) program and 'Universiteit Gent-Geconcerteerde Onderzoeks Acties' (GOA).

1. Angus DC. The search for effective therapy for sepsis: back to the drawing board? JAMA 2011; 306: 2614-2615

2. Harrois A, Huet O, Duranteau J. Alterations of mitochondrial function in sepsis and critical illness. Curr Opin Anaesthesiol 2009; 22: 143-149.

3. Hernandez G, Bruhn A, Ince C. Microcirculation in sepsis: new perspectives. Curr Vasc Pharmacol 2013; 11: 161-169.

4. Dominguez JA, Coopersmith CM. Can we protect the gut in critical illness? The role of growth factors and other novel approaches. Crit Care Clin 2010; 26: 549-565.

5. Buchman TG, Cobb JP, Lapedes AS, Kepler TB. Complex systems analysis: a tool for shock research. Shock 2001; 16: 248-251.

6. Vandendriessche B, Peperstraete H, Rogge E, Cauwels P, Hoste E, Stiedl O et al. A Multiscale Entropy-based Tool for Scoring Severity of Systemic Inflammation. Crit Care Med 2014 (in press).

7. Levy MM, Fink MP, Marshall JC, Abraham E, Angus D, Cook D et al. 2001SCCM/ESICM/ ACCP/ATS/SIS International Sepsis Definitions Conference. Crit Care Med 2003; 31: $1250-1256$.

8. Vincent JL, Opal SM, Marshall JC, Tracey KJ. Sepsis definitions: time for change. Lancet 2013; 381: 774-775

9. Chen GY, Nunez G. Sterile inflammation: sensing and reacting to damage. Nat Rev Immunol 2010; 10: 826-837. 
10. Fink MP, Heard SO. Laboratory models of sepsis and septic shock. J Surg Res 1990; 49 186-196.

11. Taveira da Silva AM, Kaulbach HC, Chuidian FS, Lambert DR, Suffredini AF, Danner RL. Brief report: shock and multiple-organ dysfunction after self-administration of Salmonella endotoxin. N Engl J Med 1993; 328: 1457-1460.

12. Kayagaki N, Warming S, Lamkanfi M, Vande Walle L, Louie S, Dong J et al. Non-canonical inflammasome activation targets caspase-11. Nature 2011; 479: 117-121.

13. Nakahira K, Haspel JA, Rathinam VA, Lee SJ, Dolinay T, Lam HC et al. Autophagy proteins regulate innate immune responses by inhibiting the release of mitochondrial DNA mediated by the NALP3 inflammasome. Nat Immunol 2011; 12: 222-230.

14. Fitz JG. Regulation of cellular ATP release. Trans Am Clin Climatol Assoc 2007; 118 199-208.

15. Labasi JM, Petrushova N, Donovan C, McCurdy S, Lira P, Payette MM et al. Absence of the P2X7 receptor alters leukocyte function and attenuates an inflammatory response. $J$ Immunol 2002; 168: 6436-6445.

16. Pelegrin $P$, Barroso-Gutierrez $C$, Surprenant $A$. P2X7 receptor differentially couples to distinc release pathways for IL-1beta in mouse macrophage. J Immunol 2008; 180: 7147-7157.

17. He Y, Franchi L, Nunez G. TLR. agonists stimulate Nlrp3-dependent IL-1beta production independently of the purinergic P2X7 receptor in dendritic cells and in vivo. $J$ Immunol 2013; 190: 334-339.

18. Lazarowski ER, Boucher RC, Harden TK. Mechanisms of release of nucleotides and integration of their action as P2X-and P2Y-receptor activating molecules. Mol Pharmacol 2003: 64: 785-795.

19. Lecut C, Faccinetto C, Delierneux C, van Oerle R, Spronk HM, Evans RJ et al. ATP-gated $\mathrm{P} 2 \mathrm{X} 1$ ion channels protect against endotoxemia by dampening neutrophil activation. J Thromb Haemost 2012; 10: 453-465.

20. Vanden Berghe T, Demon D, Bogaert P, Vandendriessche B, Goethals A, Depuydt B et al. Simultaneous targeting of Interleukin-1 and -18 is required for protection against inflammatory and septic shock. Am J Resp Crit Care Med 2014; 189: 282-291.

21. Schroder K, Tschopp J. The Inflammasomes. Cell 2010; 140: 821-832.

22. Duprez L, Takahashi N, Van Hauwermeiren F, Vandendriessche B, Goossens V, Vanden Berghe T et al. RIP kinase-dependent necrosis drives lethal systemic inflammatory response syndrome. Immunity 2011; 35: 908-918.

23. Lamkanfi M, Walle LV, Kanneganti TD. Deregulated inflammasome signaling in disease. Immunol Rev 2011; 243: 163-173.

24. Gitter AH, Bendfeldt K, Schulzke JD, Fromm M. Leaks in the epithelial barrier caused by spontaneous and TNF-alpha-induced single-cell apoptosis. Faseb J 2000; 14: 1749-1753.
25. Piguet PF, Vesin C, Donati Y, Barazzone C. TNF-induced enterocyte apoptosis and detachment in mice: Induction of caspases and prevention by a caspase inhibitor, ZVAD-fmk. Lab Invest 1999; 79: 495-500.

26. Wu ZQ, Han XD, Wang Y, Yuan KL, Jin ZM, Di JZ et al. Interleukin-1 receptor antagonist reduced apoptosis and attenuated intestinal mucositis in a 5-fluorouracil chemotherapy model in mice. Cancer Chemother Pharmacol 2011; 68: 87-96.

27. Farhadi A, Banan A, Fields J, Keshavarzian A. Intestinal barrier: an interface between health and disease. J Gastroenterol Hepatol 2003; 18: 479-497.

28. Johansson ME, Larsson JM, Hansson GC. The two mucus layers of colon are organized by the MUC2 mucin, whereas the outer layer is a legislator of host-microbial interactions. Proc Natl Acad Sci USA 2011; 108: 4659-4665.

29. Williams M, Jarvis MF. Purinergic and pyrimidinergic receptors as potential drug targets. Biochem Pharmacol 2000; 59: 1173-1185.

30. Hohenegger M, Matyash M, Poussu K, Herrmann-Frank A, Sarkozi S, Lehmann-Horn F et al. Activation of the skeletal muscle ryanodine receptor by suramin and suramin analogs. Mol Pharmacol 1996; 50: 1443-1453.

31. Zhang YL, Keng YF, Zhao Y, Wu L, Zhang ZY. Suramin is an active site-directed reversible, and tight-binding inhibitor of protein-tyrosine phosphatases. J Biol Chem 1998; 273: 12281-12287.

32. Cauwels A, Janssen B, Waeytens A, Cuvelier C, Brouckaert P. Caspase inhibition causes hyperacute tumor necrosis factor-induced shock via oxidative stress and phospholipase A2. Nat Immunol 2003; 4: 387-393.

33. Bultinck $\mathrm{J}$, Brouckaert $\mathrm{P}$, Cauwels $\mathrm{A}$. The in vivo contribution of hematopoietic cells to systemic TNF and IL-6 production during endotoxemia. Cytokine 2006; 36 : 160-166.

34. Landegren U. Measurement of cell numbers by means of the endogenous enzyme hexosaminidase. Applications to detection of lymphokines and cell surface antigens. J Immunol Methods 1984; 67: 379-388.

(i) (3) $\odot$ Cell Death and Disease is an open-access journal (ay No published by Nature Publishing Group. This work is licensed under a Creative Commons Attribution-NonCommercialNoDerivs 3.0 Unported License. To view a copy of this license, visit http://creativecommons.org/licenses/by-nc-nd/3.0/ 